\title{
Adaptive Video Transmission for OFDMA Systems
}

\author{
Guang-Hua Yang, Dongxu Shen and Victor O. K. Li \\ Department of Electrical and Electronic Engineering \\ The University of Hong Kong, Pokfulam Road, Hong Kong, China \\ Email: \{ghyang, dxshen, vli\}@eee.hku.hk
}

\begin{abstract}
An adaptive framework is proposed for multi-user video transmission over orthogonal frequency division multiple access (OFDMA) systems. Utilizing the channel knowledge, a twostep heuristic sub-carrier assignment algorithm is proposed to achieve unequal error protection for the video data. The approach also solves the fairness issue among different users that may be caused by varying channel quality on different sub-carriers. Meanwhile, multi-user channel gain is also achieved. The proposed framework significantly improves the video transmission quality with little extra computational complexity and system overhead.
\end{abstract}

\section{INTRODUCTION}

Providing high-quality video services is an important task for wireless broadband communication systems. Unequal error protection (UEP) is effective in improving video transmission quality over error prone channels by providing better protection or better transmission opportunity to the video data with higher degree of importance [1]-[4]. On the other hand, OFDMA is considered one of the key technologies for future multi-user broadband wireless access (BWA).

Traditional scheduling schemes in OFDMA only consider the multi-user gain but ignore the different importance of data in sub-carrier assignment. In this work, we propose an adaptive video transmission framework based on a two-step heuristic sub-carrier assignment algorithm with the consideration of the unequal degrees of importance within the video data. Video data is divided into a high priority (HP) and a low priority (LP) layer based on their relative importance. All the sub-carriers are then indexed by the channel quality. In the first step of the algorithm, HP data of all users is allocated high quality (HQ) sub-carriers, and LP data is assigned the remaining low quality (LQ) sub-carriers. On the other hand, the channel quality is essentially identical among HP data of different users, but the channel quality degrades with the order of assignment for the LP data. Thus there is a short-term fairness problem for the LP data. We then propose an alternative algorithm to resolve this issue. The second allocation step is divided into several rounds: in each round only one sub-carrier is assigned to a user, and the allocation continues until all sub-carriers are assigned to users.

The rest of the paper is organized as follows. In Section II, we introduce the system model and present the framework of the adaptive video transmission scheme based on the twostep sub-carrier assignment algorithm. Simulation results are presented in Section III. Conclusion is given in Section IV.

\section{AdAPtive Video TRANSMisSiOn FOR OFDMA SYSTEMS}

\section{A. System Model}

We consider the downlink (base station to mobile terminals) data transmissions in a system consisting of one central base station (BS) and $K$ mobile terminals located randomly around the BS. In one OFDM symbol, there are $N$ sub-carriers for data transmission. Each sub-carrier can only be assigned to one mobile terminal. Through the control channel, the sub-carrier allocation map is sent to all mobile users.

At time $t$, let $h_{n, k}(t)$ and $\delta_{n, k}(t)$ denote the channel gain and noise level of sub-carrier $k$ with respect to user $n$. Here $h_{n, k}(t)$ incorporates the effects of path loss, shadowing and fading. Define the channel to noise ratio $(\mathrm{CNR})$ for user $n$ on sub-carrier $k$ as

$$
C N R_{k, n}=\frac{h_{n, k}^{2}(t)}{\delta_{n, k}^{2}(t)} .
$$

Then the signal to noise ratio (SNR) can be derived from $C N R$ as

$$
S N R_{n, k}=p_{n, k}(t) \times C N R_{k, n},
$$

where $p_{n, k}$ is the transmission power of sub-carrier $k$. The bit error rate (BER) is determined by $S N R_{n, k}$ and the employed modulation scheme. We assume all the sub-carriers have the same modulation scheme and the same transmission power. We assume the channel information for all users is known at the BS.

\section{B. Heuristic Sub-carrier Assignment Scheme}

In [5], a simple heuristic channel assignment algorithm is proposed. The idea is to simply assign the best available subcarriers in terms of $C N R$ to each user sequentially. Then the assignment order is rotated to achieve a good fairness among users. The algorithm is summarized in Algorithm 1.

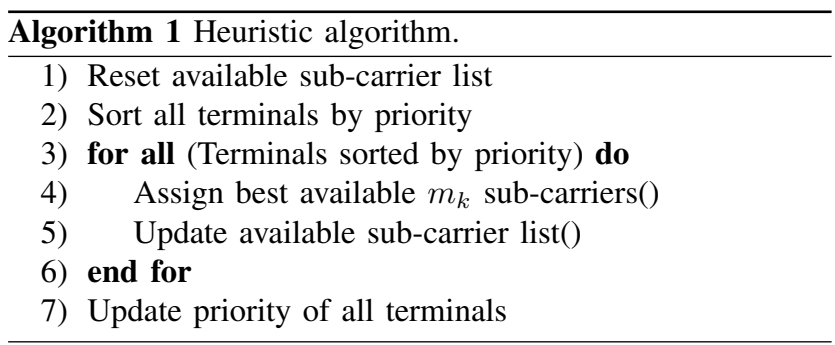




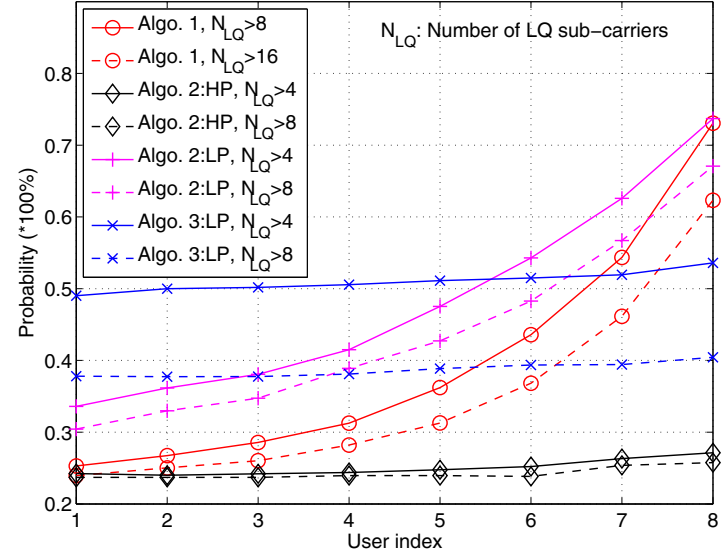

Fig. 1. Probability of being assigned excessive number of low quality subcarriers.

In Algorithm 1, integers 1 to $K$ are assigned to $K$ users as the initial priority. At the beginning of each sub-carrier assignment cycle, all sub-carriers are set to be available. The algorithm iterates over all users sorted by their priorities. For each iteration step, the considered user is assigned the best $m_{k}$ ${ }^{1}$ available sub-carriers in terms of $C N R$ value. Then these sub-carriers are removed from the available sub-carrier pool. After the whole sub-carrier assignment iteration is finished, the priority of each user is decreased by one (reset to $K$ when it reaches 0) as the preparation for the next run.

The above heuristic algorithm suffers a fairness problem, as demonstrated by the following simulation. Here we consider an OFDMA system with 256 sub-carriers and 8 users to illustrate the deep-fading-sub-carrier problem, and all users are assigned 32 sub-carriers. Assume the average sub-carrier gain is 1 for all sub-carriers and a sub-carrier is labeled as high quality (HQ) if the instant sub-carrier gain is no less than 1 , while others are low quality (LQ) sub-carriers. With a fixed assignment order, we count the number of LQ subcarriers assigned to each user, and calculate the probabilities that the number of LQ sub-carriers exceeds a pre-defined threshold. The two circle-marked curves (Algo. 1) in Fig. 1 show the simulation results when the threshold is 8 and 16 , respectively. From the two curves, we find that Algorithm 1 has two main drawbacks. One is that the probability for the number of LQ sub-carriers exceeding the threshold is quite high (when the threshold is 16 , which equals half of the total of 32 sub-carriers, the probability is even higher than $50 \%$ for some users). The second problem is that the algorithm does not provide a good short-term fairness for different users. The probability increases sharply with the assignment order. The user in the last iteration has a much higher probability to be assigned LQ sub-carriers than the users assigned earlier. Although rotation of the sub-carrier assignment order helps to achieve long-term fairness, it does not resolve the short-term

\footnotetext{
${ }^{1} m_{k}$ can be obtained by employing a resource allocation algorithm in advance, and is not part of the considered algorithm.
}

fairness problem.

\section{Adaptive Two-step Sub-carrier Assignment}

To resolve the above mentioned problems, and more importantly to achieve UEP, we propose a new two-step subcarrier assignment algorithm. At the first step, the sub-carriers are assigned to HP data with the heuristic algorithm (Algorithm 1). Then in the second step, the remaining sub-carriers are assigned to LP data with the heuristic algorithm again. Algorithm 2 describes this process.

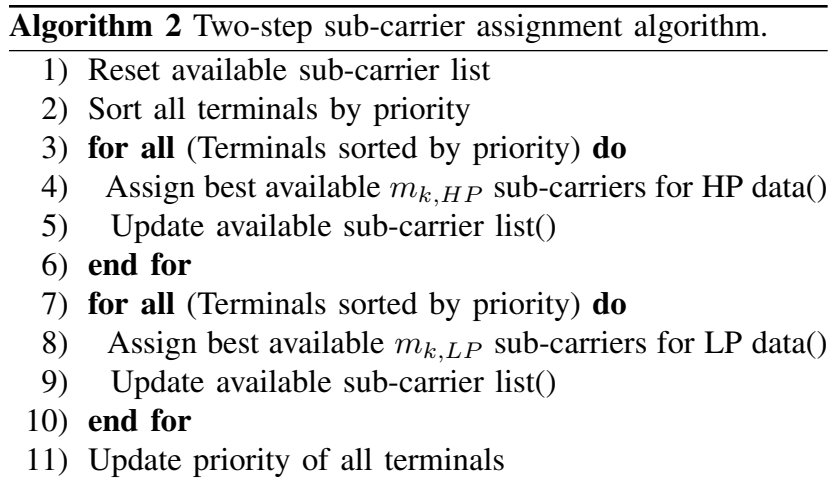

Let $m_{k, H P}$ and $m_{k, L P}$ be the number of sub-carriers for HP and LP data to be assigned to a user $k$. With Algorithm 2, HP data for all the users have a higher priority in sub-carrier assignment than the LP data. If $m_{k, H P}=m_{k, L P}$, even for the last user in a sub-carrier assignment cycle, more than half of all the sub-carriers are still available for selection. That means HP data has a relatively low probability to be transmitted over sub-carriers in deep fading, resulting in a lower error rate for HP data and a better video transmission quality.

We also use the configuration in Sec. II-B to evaluate the two-step adaptive sub-carrier assignment algorithm. The corresponding probability of LQ sub-carrier number exceeding the threshold for both HP and LP data of different users are also shown in Fig. 1, as denoted by Algo. 2. The probability of employing an LQ sub-channel for HP data is quite low and almost the same for different users, while the probability for LP data has the same trend as the heuristic algorithm in Sec. II-B. Therefore, the two-step adaptive sub-carrier assignment algorithm provides different levels of transmission opportunities for data with different levels of importance.

\section{Adaptive Two-step Sub-carrier Assignment with Short-term Fairness}

It can be observed from Fig. 1 that LP data for the users with higher index are more likely to experience LQ sub-carriers. On the other hand, the curve for the HP sub-carriers is almost flat, which means Algorithm 2 ensures the short-term fairness for HP data but not for LP data. This is because most of the HQ sub-carriers are not in deep fades. On the other hand, when the sub-carriers for LP data are assigned, the user index becomes important, because the user assigned in a later stage has a higher probability to be assigned with sub-carriers in deep fades. 
Thus we modify the assignment algorithm for the LP data: only one sub-carrier is assigned to a user during one round of sub-carrier assignment, as shown by the pseudo codes in Algorithm 3.

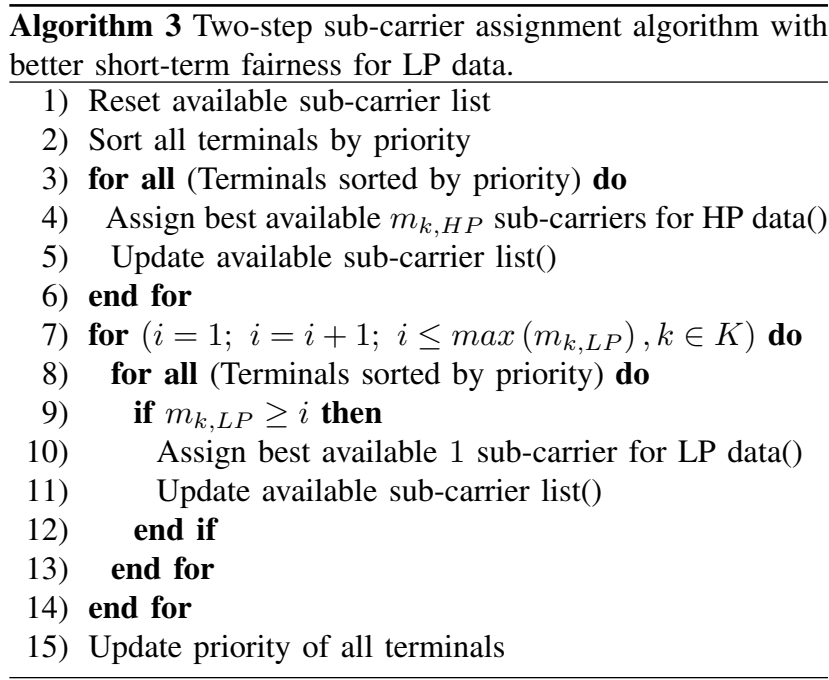

With the same configuration as in Sec. II-B, the corresponding probability of LQ sub-carrier number exceeding the threshold for LP data of different users are calculated and also shown in Fig. 1 (Algo. 3). From the figure, we can find that the curve is flat, which means the assignment is fair among users.

Algorithm 3 achieves a better fairness performance over Algorithm 2, but with a higher computational overhead and higher complexity for the LP data. Since LP data has limited contribution to the final reconstructed video quality, the fairness for LP data has limited effect on the system performance. This point will be demonstrated by simulation in Sec. III.

\section{E. System Framework}

Fig. 2 depicts the system framework of the proposed transport prioritization scheme. At the base station, video streams for different users are layer-coded into two data streams and buffered in two separate FIFO queues. With the above subcarrier assignment algorithm, sub-carriers are allocated to different users and a sub-carrier allocation vector (SCAV) is generated to indicate the allocation. Data is sent using standard OFDM techniques on sub-carriers according to SCAV, which is sent to all users by broadcasting over a control channel. At the receiver, standard detection and decoding algorithms are performed to decode the whole OFDM symbol, and the layered video data for a certain user is extracted from the symbol according to SCAV.

\section{Simulation Results}

\section{A. Simulation Setup}

In the performance comparison, the algorithms in Sec. IIB, Sec. II-C and Sec. II-D are denoted as Algo. 1, Algo. 2, and Algo. 3, respectively. Besides the proposed adaptive twostep sub-carrier assignment with UEP, two other systems are

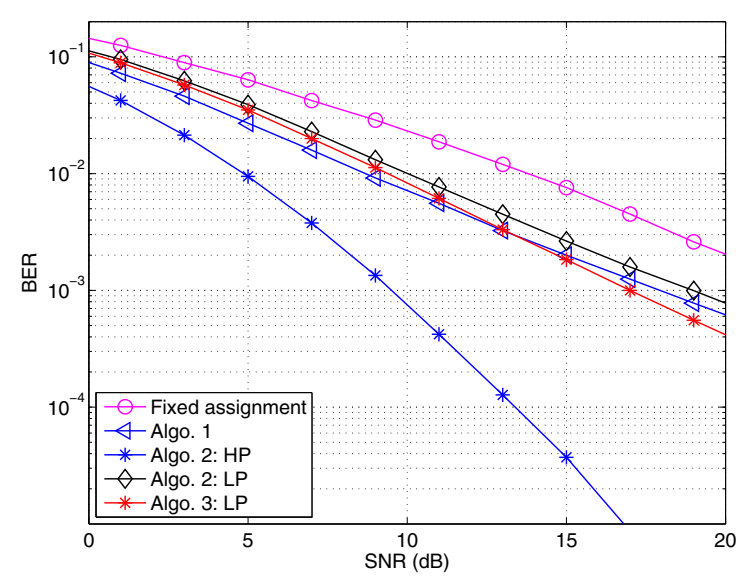

Fig. 3. Comparison of bit error rate.

also simulated for comparison. One is an OFDMA system with fixed sub-carrier allocation and no UEP. The other is an OFDMA system with heuristic sub-carrier allocation and no UEP. The standard video sequence "Mobile" is used as the video source.

In the simulation, we consider an OFDMA system with 256 sub-carriers. Four users are randomly located around the BS, and performance results are averaged over all locations. One video transmission link is set up from the BS to each of the users. The delay profile of indoor wideband channel model B, provided in the ITU-R recommendation [6], is adopted for the simulation of uncorrelated multipath Rayleigh fading channels. The Doppler frequency is fixed at $100 \mathrm{~Hz}$.

The standard video sequence "Mobile" is used as the video source. The sequence is in the common intermediate format (CIF) with a resolution of $352 \times 288$, and color format of $4: 2$ : 0. MPEG-2 [7] is employed to encode the sequence at 30 frames per second with a bit rate of $4 \mathrm{Mbps}$. Data partitioning is employed to separate the compressed video into HP and LP data sub-streams, each at about 2 Mbps. A simple video error concealment scheme is employed at the decoder to replace the undecodable macro-block or frame with the last correctly decoded one. The peak signal-to-noise ratio (PSNR) is employed to evaluate the reconstructed video quality.

\section{B. BER Comparison}

We compare the BER of the three considered systems and show the simulation results in Fig. 3. Since the HP (LP) data for different users has similar BER performance, we only plot one curve for HP (LP) data. As expected, due to the multi-user gain, the heuristic algorithm achieves a lower BER than the fixed sub-carrier assignment scheme.

For Algo. 2, HP data achieves much better BER performance than that of the heuristic algorithm. For the LP data, there is a constant SNR loss of about $1.5 d B$ with respect to the heuristic scheme. Therefore, the BER of the LP data is sacrificed in exchange for better BER for the HP data.

For the performance of Algo. 3, only the LP curve is plotted, because the HP BER performance remains unchanged with 


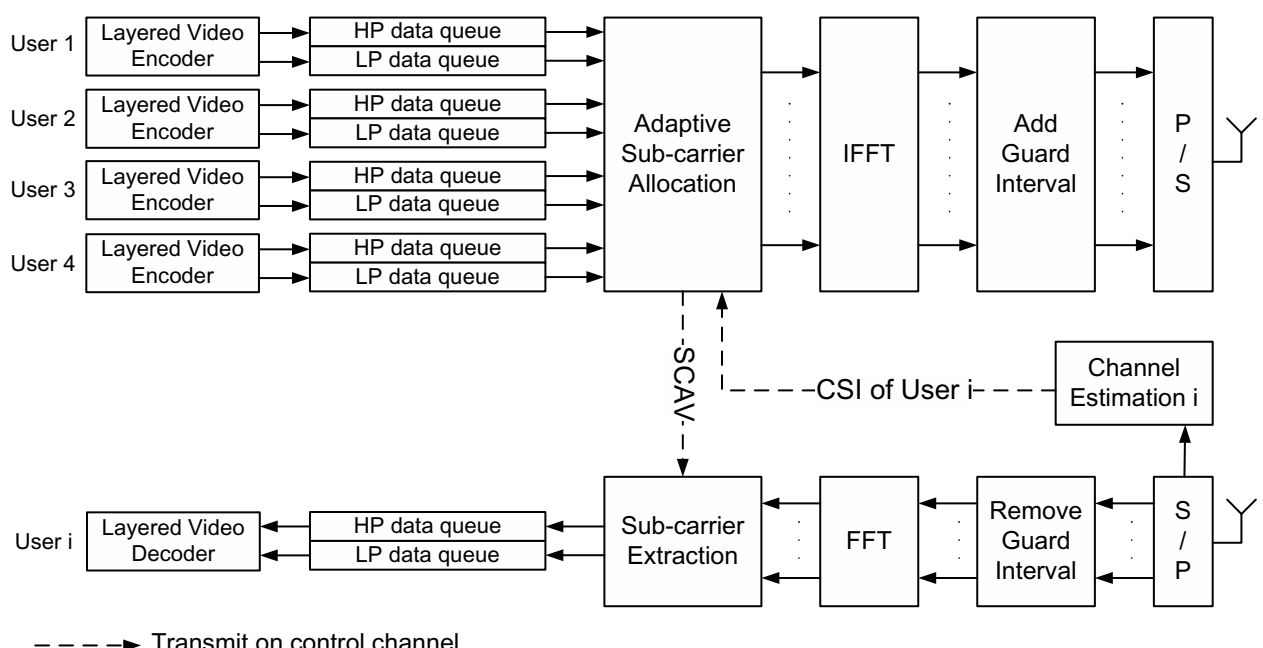

SCAV: Sub-Carrier Allocation Vector, CSI: Channel State Information, P/S: Parallel to Serial Transformation

Fig. 2. Framework of the proposed adaptive video transmission over OFDMA systems.

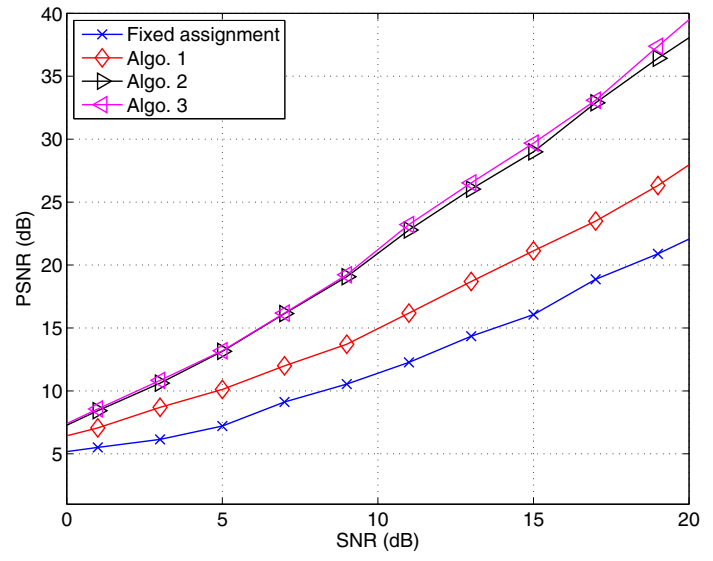

Fig. 4. Comparison of reconstructed video quality.

respect to that of Algo. 2. The performance for the LP data is clearly better than that of Algo. 2, with more than $2 d B$ SNR gain at a SNR of $20 d B$.

\section{Performance of Adaptive Video Transmission}

Since HP data is much more important to the final video quality than LP data, the BER reduction of HP data compensates for the increased BER of LP data, resulting in a better reconstructed video quality. In Fig. 4, we compare the PSNR performance of the three considered systems.

The heuristic scheme (Algo. 1, and without UEP) outperforms the fixed assignment scheme with about $4 d B$ PSNR gain, because of the multi-user diversity gain. Algo. 2 and Algo. 3 are both UEP schemes, and they achieve consistently better performance than the simple heuristic scheme Algo. 1 . The PSNR gain is even larger with the increase of SNR. From Fig. 4, we find that at $S N R=20 d B$, the two proposed schemes have about $10 d B$ and $15 d B$ PSNR gain over the heuristic scheme and the fixed sub-carrier assignment scheme, respectively.

On the other hand, the performance gap between Algo. 2 and Algo. 3 is negligible. Algo. 3 only outperforms Algo. 2 slightly at SNR's higher than $10 d B$. This is because the lower BER achieved by Algo. 3 for the LP data only contributes insignificantly to the final PSNR performance. Since Algo. 3 involves multiple rounds of sub-carrier assignment for the LP data, this insignificant performance gain is not worth the extra complexity when SNR is low. Therefore, we conclude that Algo. 2 is a better choice.

\section{CONCLUSION}

In this paper, a two-step adaptive sub-carrier assignment algorithm is proposed to exploit the multi-user gain and provide transmission differentiation for data with different degrees of importance. Meanwhile, the fairness among different users and multi-user channel gain are also achieved. The new scheme achieves significantly better video transmission quality with little extra computational complexity and overhead.

\section{REFERENCES}

[1] L. Hanzo and J. Streit, "Adaptive low-rate wireless video phone schemes," IEEE Trans. Circuits Syst. Video Technol., vol. 5, no. 4, pp. 305-318, August 1995.

[2] G.-H. Yang, D. Shen, and V. O. K. Li, "UEP for video transmission in space-time coded OFDM systems," in Proc. IEEE INFOCOM'04, Hong Kong, P. R. China, March 2004.

[3] — "Unequal error protection for MIMO systems with a hybrid structure," in Proc. IEEE ISCAS 2006, Island of Kos, Greece, May 2006.

[4] - "Adaptive video streaming over multi-channel ad hoc networks," in Proc. IEEE Globecom'2006, San Francisco, US, November 2006.

[5] J. Gross, H. Karl, F. Fitzek, and A. Wolisz, "Comparison of heuristic and optimal subcarrier assignment algorithms," in Proc. of Intl. Conf. on Wireless Networks (ICWN), Las Vegas, NV, USA, June 2003.

[6] Guidelines for evaluation of radio transmission technologies for IMT2000, ITU-R M.1225, ITU-R Recommendation.

[7] Information Technology - Generic Coding of Moving Pictures and Associated Audio Information: Video, ITU-T Rec. H.262 - ISO/IEC 13818-2, July 1995 . 